American Journal of Applied Sciences 7 (1): 38-43, 2010

ISSN 1546-9239

(C) 2010 Science Publications

\title{
Bacterial Profile and Antimicrobial Resistance to Commonly Used Antimicrobials in Intra-Abdominal Infections in Two Teaching Hospitals
}

\author{
${ }^{1}$ Gholamreza Sepehri, ${ }^{2}$ Hamid Zeinali Nejad, ${ }^{3}$ Ehsan Sepehri and ${ }^{4}$ Saeideh Razban \\ ${ }^{1}$ Neuroscience Research Center, Department of Physiology and Pharmacology, \\ Kerman University of Medical Sciences, Kerman, Iran \\ ${ }^{2}$ Physiology Research Center, Department of Surgery, \\ Kerman University of Medical Sciences, Kerman, Iran \\ ${ }^{3}$ Medical School, Tehran University of Medical Sciences, Tehran, Iran \\ ${ }^{4}$ Veterinary Medicine School, Bahonar University, Kerman, Iran
}

\begin{abstract}
Problem statement: Intra-abdominal infections are associated with significant morbidity and mortality. The resulting infection is typically polymicrobial and comprised of both aerobic and anaerobic microbes, which need systemic antimicrobial therapy. Since the bacteriology and antimicrobial susceptibility of postoperative intra abdominal infections were not determined in Iran, so this study was performed to evaluate the antimicrobial susceptibility patterns among aerobic bacteria isolated from post-operative intra-abdominal selected samples in 2 teaching hospitals in Kerman, Iran. Approach: the peritoneal sample of 174 patients which undergone abdominal surgery were cultured by routine microbiological methods for aerobic microorganisms. Antimicrobial susceptibility testing was performed to 4 commonly used antibacterials (Ampicillin, Cefazolin, Gentamicin and Ciprofloxacin) using disc diffusion method according to the NCCLS guidelines. Results: Escherichia coli (E. coli) was the most frequent microorganism which was isolated from $70.6 \%$ of peritoneal cultures, followed by Klebsiella pneumonia (13.7\%), Pseudomonas aeruginosa (10.8\%) and Proteus mirabilis $(4.9 \%)$. E. coli which was the most common isolate was highly susceptible to ciprofloxacin $(84.6 \%)$ and gentamicin $(76.9 \%)$. The resistance rate of E. coli isolates to ampicillin was very high, i.e, $80.8 \%$ of $E$. coli isolates were resistant to ampicillin. The resistance rate for pseudomonas and Klebsiella spp. to commonly used antimicrobials varied from $25 \%$ to ciprofloxacin to $100 \%$ to ampicillin. Conclusion: E. coli was the most common isolate in post-operative peritoneal cultures. The results showed the relatively high resistance rate of the isolated microorganisms to commonly used antimicrobials, especially to ampicillin. So the choice of antimicrobial therapy must be based on the susceptibility tests and also take into account the risk of inadequate and in appropriate antimicrobial therapy and emergence of bacterial resistance to commonly used antimicrobials.
\end{abstract}

Key words: Intra-abdominal infections, antimicrobial resistance, abdominal surgery

\section{INTRODUCTION}

Abdominal infections are associated with significant morbidity and mortality. Nearly all bacteria causing abdominal infections are derived from the endogenous flora of the alimentary tract (Laterre et al., 2006). The resulting infection is typically polymicrobial and comprised of both aerobic and anaerobic microbes. They can be classified by their severity as uncomplicated and complicated or by their origin as community or hospital acquired (Mazuski et al., 2002a; Onderdonk et al., 1990). Complicated infections are those that require both surgical or radiological drainage procedures and antimicrobial therapy.Antibiotics play an important role in prevention and treatment of intraabdominal infections (Blot and De Waele, 2005; Driscoll et al., 2007; Dellinger et al., 1994).

Intra-abdominal infections are common causes of hospitalization with approximately 2 million intraabdominal procedures performed each year in the USA (Mazuski et al., 1990).

The aerobes isolated include E. coli, usually the major isolate, enterococci, viridans streptococci, other Enterobacteriaceae, Proteus spp. and occasionally Pseudomonas aeruginosa, Serratia spp. and Acinetobacter spp. (the latter three are often associated

Corresponding Author: Gholamreza Sepehri, Neuroscience Research Center, Department of Physiology and Pharmacology, Kerman University of Medical Sciences, Kerman, Iran 
Am. J. Applied Sci., 7 (1): 38-43, 2010

with nosocomial infections). The anaerobes are predominantly the Bacteroides species and Bifidobacterium species (Laterre et al., 2006; Goldstein and Snydman, 2004; Pieracci and Barie, 2007a; Kroenke, 1985).

Antimicrobial therapy poses an important clinical challenge because of the diverse bacteriology of complicated intra-abdominal infections and the emergence of bacterial resistance. In general, selection of an empiric agent or combination regimen must be directed at providing reliable activity against $E$. coli, other gram negative facultative bacteria and $B$ fragilis (Mazuski et al., 2002a; Guembe and 2008; Solomkin et al., 2003; Marshall, 2004).

Many other factors influence the selection of an antimicrobial agent, including its potential to induce bacterial resistance, its risk of hypersensitivity, its overall tolerability, its dosing frequency and its cost. Accordingly, the search continues for an effective antimicrobial regimen that has activity against resistant pathogens, a minimal risk of side effects, a convenient dosing schedule and potential cost benefits (Bohnen et al., 1992a; 1992b; Goldstein and Snydman, 2004; Hasper et al., 2009; Solomkin et al., 2003; Pieracci and Barie, 2007b).

Several intravenous antibiotics have been investigated, as monotherapy or as part of a combination regimen, for the management of patients with intra-abdominal infections (Weigelt, 2007). The old standard of care involved double or tripleantimicrobial therapy (e.g., aminoglycoside/betalactam/clindamycin) to provide coverage against an array of potential pathogens (Weigelt, 2007). In recent years, monotherapy with imipenem/cilastatin (Primaxin) has become the new gold standard because of its broad spectrum of activity against anticipated pathogens and its relative safety and ease of use. In addition to imipenem/cilastatin contemporary agents with documented efficacy include cefoxitin, ampicillin/sulbactam, ticarcillin clavulanate (Timentin) and piperacillin/tazobactam (Zosyn) but the emergence of multidrug-resistant Enterobacteriaceae and other gram-negative bacilli have become a growing problem (Mazuski et al., 2002a; Goldstein and Snydman. 2004; Solomkin et al., 2003; Weigelt, 2007; Mazuski et al., 2002b).

Antibiotics are among the most misused drugs in Iran and are prescribed inappropriately for outpatients which causes the emergence of bacterial resistance to commonly used antimicrobials (Sepehri and Meimandi, 2005). Since the bacteriology and antimicrobial susceptibility of postoperative intra abdominal infections were not determined in Iran, so this study was performed to evaluate the antimicrobial susceptibility patterns among aerobic bacteria isolated from post-operative intra-abdominal selected samples in 2 teaching hospitals in Kerman, Iran.

\section{MATERIALS AND METHODS}

This cross-sectional study was conducted in 2008 in two teaching hospitals of Kerman University of Medical Sciences. Kerman (the center of Kerman province) is situated $1,000 \mathrm{Km}$ from Tehran in south of Iran having around 600,000 residents.

The peritoneal samples of 174 patients which undergone abdominal surgery were cultured by routine microbiological methods for aerobic microorganisms.

Samples were streaked on two plates that consist of blood agar supplemented with $5 \%$ defibrinated sheep blood and Eosin Methylene Blue (EMB) agar. Plates were incubated aerobically at $37^{\circ} \mathrm{C}$ for $48 \mathrm{~h}$. Based on colony morphology, positive cultures were Gram stained and Gram-positive organisms were subcultured on blood agar plates (Padtan Teb Co, IRAN) Gramnegative rods were subcultured on MacConkey agar (Padtan Teb Co, IRAN). Organisms were identified using standard methods and API Identification System. Gram positive and gram-negative isolates bacterial sensitivity to commonly used antimicrobials (Ampicillin, Cefazolin, Gentamicin, Ciprofloxacin) were investigated by disk diffusion method using NCCLS guidelines (Ginocchio, 2002; Zapantis et al., 2005).

Data were entered and analyzed by Stata v. 8. and results were reported as the type and percentage of microbial isolations and also the percentage of microbial susceptibility and resistance to commonly used antimicrobials.

\section{RESULTS}

A total of 174 patients were hospitalized for Intraabdominal infections during the first 9 months of 2008 in two teaching hospitals in Kerman City. 96 $(55.1 \%)$ of patients were men and $78(44.9 \%)$ were women which showed significant difference $(p<0.05)$. The results of microbiological cultures showed that no aerobic microbial isolates was found in $41.4 \%$ of patients. Aerobic microorganisms were isolated in 102 (58.6\%) patients. Escherichia coli 
(E. coli) was the most commonly cultured microorganisms isolated from peritoneal samples (70.6\%) followed by Klebsiella pneumonia (13.7\%), Pseudomonas aeruginosa (10.8\%) and Proteus mirabilis $(4.9 \%)$ (Table1). The co-infection with two bacterial species (including E. coli and Pseudomonas aeruginosa) was seen in $7(6.9 \%)$ of peritoneal samples. The resistance rates to commonly used antimicrobials in isolated bacteria from peritoneal samples varied from $15.4 \%$ for ciprofloxacin to $100 \%$ for ampicillin (Table 2). E. coli isolates were highly sensitive to ciprofloxacin $(84.6 \%)$ but showed high resistance rate $(80.8 \%)$ to ampicillin (Table 2 ).

Also Pseudomonas aeruginosa isolates showed high sensitivity to ciprofloxacin and gentamicin (75\%) and high resistance rate to cefazolin (75\%) and ampicillin (100\%) (Table 2).

The resistance rate of Klebsiella pneumonia to commonly used antimicribials varied from $40 \%$ to ciprofloxacin and gentamicin to $80 \%$ for ampicillin (Table 2). The results of this study showed that $64.3 \%$ of isolates were resistant to at least 2 antibiotics and $7.1 \%$ of isolates were resistant to all 4 used antimicrobials (Table 3).

Table 1: Type of microorganisms isolated from peritoneal samples in 174 patients with intra-abdominal operations in Kerman teaching hospitals in 2008, Kerman, Iran

\begin{tabular}{lcc}
\hline Microbial agent & Number of isolates & Isolates (\%) \\
\hline Escherichia coli & 72 & 70.6 \\
Klebsiella pneumonia & 14 & 13.7 \\
Pseudomonas aeruginosa & 11 & 10.8 \\
Proteus mirabilis & 5 & 4.9 \\
Total & 102 & 100.0 \\
Mixed isolates & 7 & 6.9 \\
\hline
\end{tabular}

Table 2: Resistance rate to commonly used antimicrobials in 174 patients with intra-abdominal operations in Kerman teaching hospitals in 2008, Kerman, Iran

\begin{tabular}{llll}
\hline & $\begin{array}{l}\text { Resistance rate } \\
\text { Escherichia coli } \\
(\mathrm{n}=72)\end{array}$ & $\begin{array}{l}\text { Kleb. Pneumonia } \\
(\mathrm{n}=14)\end{array}$ & $\begin{array}{l}\text { Pseudo. Aeruginosa } \\
(\mathrm{n}=11)\end{array}$ \\
Antimicrobial & 15.4 & 40 & 25 \\
\hline Ciprofloxacin & 15.4 & 60 & 75 \\
Cefazolin & 43.2 & 80 & 100 \\
Ampicillin & 80.8 & 40 & 25 \\
Gentamicin & 23.1 & & \\
\hline
\end{tabular}

Table 3: Frequency of microbial isolates co- resistance to commonly used antimicrobials in 174 patients with intra-abdominal operations in Kerman teaching hospitals in 2008, Kerman, Iran

\begin{tabular}{lcl}
\hline $\begin{array}{l}\text { Co- resistance to } \\
\text { antimicrobials }\end{array}$ & $\begin{array}{l}\text { Resistant } \\
\text { isolates (n) }\end{array}$ & $\begin{array}{l}\text { Resistant } \\
\text { isolates (\%) }\end{array}$ \\
\hline One & 5 & 17.9 \\
Two & 18 & 64.3 \\
Three & 3 & 10.7 \\
Four & 2 & 7.1 \\
\hline
\end{tabular}

\section{DISCUSSION}

The results of this study showed that E. coli was most frequent microorganism isolated from peritoneal samples in patients undergone abdominal surgery. Others also reported that $E$. coli is the most commonly isolated aerobic microorganism isolated from intraabdominal infections (Snydman, 2004; Hasper et al., 2009; Pieracci and Barie, 2007b; Weigelt, 2007).

Klebsiella pneumonia, Pseudomonas aeruginosa and Proteus mirabilis were other microorganisms which were isolated in a few percents of patients which are in complete agreement with other investigators reports (Snydman, 2004; Hasper et al., 2009; Mazuski, 2007; Paterson et al., 2005).

Pseudomonas aeruginosa isolation in intraabdominal surgery patients which is one of the potential pathogens could be of important concern, because it is a major cause of nosocomial infections and this organism shows a remarkable capacity to resist antibiotics (Driscoll et al., 2007; Montravers et al., 2009; Obritsch et al., 2005). Infection with Pseudomonas aeruginosa is typically observed in high-risk patients such as those with lateonset nosocomial infection and those who have received previous antimicrobial therapy, undergone recurrent surgeries, or both. In constrast, patients with early-onset health care-associated or communityacquired infections have a low prevalence of Pseudomonas aeruginosa (Mazuski et al., 2002b; Weigelt, 2007; Mazuski, 2007; Hasper et al., 2009).

Anaerobic microorganisms, including Bacteroids (especially Bacteroides fragilis) are among the most commonly isolated microorganisms and are isolated in more than $80 \%$ of complicated intra-abdominal infections, however, in this study only the aerobic microorganisms were isolated and this is one of the limitations of present study (Guembe et al., 2008; Baron et al., 1992; Bennion et al., 1990; Goldstein et al., 2000).13,26-28

Also others reported the isolation of Enterobacter, Staphylococcus aureus, Acinetobacter in small portion of intra-abdominal samples (Hasper et al., 2009; Guembe et al., 2008; De Vera and Simmons, 1996; Smith et al., 1999).

The prevalence of positive abdominal cultures in this study was about 58 percent which is in the range of other reported values $(28.3 \%$ in uncomplicated intraabdominal infections to $69 \%$ in complicated intraabdominal infections) (Goldstein and Snydman, 2004).

The susceptibility to commonly used antimicrobials showed a relatively high resistance rate $(80.8 \%)$ of $E$. coli to ampicillin which is clinically 
important and may indicate inappropriate use of antimicrobials in teaching hospitals in Iran, however, E. coli was very sensitive to ciprofloxacin (84.6\%) and gentamicin $(76.9 \%)$ which is in agreement with the results of other investigators in other parts of the world (80-97\%) (Goldstein and Snydman. 2004; Goldstein and Snydman. 2004; Paterson et al., 2005).

Koksal et al. (2009) reported that the resistance rate to ciprofloxacin and gentamicin in Turkey was 57.6 and $38 \%$ respectively, which is much higher than the resistance rate in our study.

The E. coli sensitivity to cefazolin $(56.8 \%)$ was significantly higher than other studies (Goldstein and Snydman, 2004; Goldstein and Snydman, 2004; Paterson et al., 2005).

Klebsiella pneumonia was relatively resistant to commonly used antimicrobials used for treatment of intra-abdominal infections. Our data are in agreement with several studies in other parts of the world showing an increasing portion of resistant isolates of Klebsiella pneumonia (Koksal et al., 2009; Jouini et al., 2009; Schito et al., 2009). Klebsiella pneumonia sensitivity to ciprofloxacin varied from $80.7 \%$ in Latin America to $91.4 \%$ in Europe countries which is comparable to the sensitivity rate in this study $(75 \%)$, however, Klebsiella pneumonia sensitivity to ampicillin was $20 \%$ which much lower than the reported value in middle east (75.6\%) and USA (91.3\%) (Paterson et al., 2005).

Pseudomonas aeruginosa isolates evaluated by this study showed very high resistance to beta lactam antibiotics (ampicillin and cefazolin). The best coverage against these isolates was obtained with ciprofloxacin and gentamicin. These data are in complete agreement with other studies which shows an increase in resistance rate to commonly used antimicrobials (Driscoll et al., 2007, Montravers et al., 2009; Obritsch et al., 2005; De Francesco et al., 2007; Mesaros et al., 2007). Also the results of this study showed that $64.3 \%$ of isolates were resistant to at least 2 antibiotics and $7.1 \%$ of isolates were resistant to all 4 used antimicrobials.

\section{CONCLUSION}

In summary, the results of this study showed that E. coli was the most common isolate in post-operative peritoneal cultures. The sensitivity to commonly used antimicrobials varied and $E$. coli showed the highest sensitivity to ciprofloxacin and gentamicin and ampicillin showed the lowest antibacterial activity against microbial isolates from post-operative peritoneal cultures. The high antimicrobial resistance to commonly used antimicrobials could profoundly affect the choice of therapeutic agents. So the choice of antimicrobial therapy must be based on culture growth and antibacterial sensitivity tests and also take into account the risk of inadequate and inappropriate antimicrobial therapy and emergence of bacterial resistance to commonly used antimicrobials.

\section{ACKNOWLEDMENT}

This study was carried out in Physiology Research Center of Kerman University of Medical Sciences. The funding of this study was provided by research deputy of Kerman University of Medical Sciences, Kerman, Iran.

\section{REFERENCES}

Baron, E.J., R. Bennion, J. Thompson, C. Strong and P. Summanen et al., 1992. A microbiological comparison between acute and complicated appendicitis. Clin. Infect. Dis., 14: 227-231. http://www.ncbi.nlm.nih.gov/sites/entrez

Bennion, R.S., E.J. Baron, J.E. Thompson Jr., J. Downes and P. Summanen et al., 1990. The bacteriology of gangrenous and perforated appendicitis-revisited. Ann. Surg., 211: 165-171. http://www.ncbi.nlm.nih.gov/pmc/articles/PMC135 7960/?tool=pubmed

Blot, S. and J.J. De Waele. 2005. Critical issues in the clinical management of complicated intraabdominal infections. Drugs, 65: 1611-1620. PMID: 16060697

Bohnen, J.M., 1992a. Operative management of intraabdominal infections. Infect. Dis. Clin. North Am., 6: 511-523. PMID: 1431036

Bohnen, J.M., J.S. Solomkin, E.P. Dellinger, H.S. Bjornson and C.P. Page, 1992b. Guidelines for clinical care: Anti-infective agents for intra-abdominal infection. A surgical infection society policy statement. Arch. Surg., 127: 83-89. http://archsurg.amaassn.org/cgi/reprint/127/1/83

De Francesco, M.A., G. Ravizzola, L. Peroni, R. Negrini and N. Manca, 2007. Urinary tract infections in Brescia, Italy: Etiology of uropathogens and antimicrobial resistance of common uropathogens. Med. Sci. Monit., 13: 136-144. http://www.medscimonit.com/fulltxt.php?ICID=48 4100

De Vera, M.E. and R.L. Simmons., 1996. Antibioticresistant enterococci and the changing face of surgical infections. Arch. Surg., 131: 338-342. http://archsurg.ama-assn.org/cgi/reprint/131/3/338 
Dellinger, E.P., P.A. Gross, T.L. Barrett, P.J. Krause and W.J. Martone et al., 1994. Quality standard for antimicrobial prophylaxis in surgical procedures. Infectious diseases society of America. Clin. Infect. Dis., 18: 422-427. PMID: 8011827

Driscoll, J.A., S.L. Brody and M.H. Kollef, 2007. The epidemiology, pathogenesis and treatment of Pseudomonas aeruginosa infections. Drugs, 67: 351-368. PMID: 17335295

Ginocchio, C.C., 2002. Role of NCCLS in antimicrobial susceptibility testing and monitoring. Am. J. Health Syst. Pharm., 59: S7-11. http://www.ajhp.org/cgi/reprint/59/suppl_3/S7

Goldstein, E.J. and D.R. Snydman, 2004. Intraabdominal infections: Review of the bacteriology, antimicrobial susceptibility and the role of ertapenem in their therapy. J. Antimicrob. Chemother., 53: ii29-ii36. DOI: 10.1093/jac/dkh201

Goldstein, E.J., D.M. Citron, C. V. Merriam, Y. Warren and K.L. Tyrrell, 2000. Comparative in vitro activities of ertapenem (MK-0826) against 1,001 anaerobes isolated from human intra-abdominal infections. Antimicrob. Agents Chemother., 44: 2389-2394.

http://www.ncbi.nlm.nih.gov/pmc/articles/PMC900 74/pdf/ac002389.pdf

Guembe, M., E. Cercenado, L. Alcala, M. Marin, R. Insa and E. Bouza, 2008. Evolution of antimicrobial susceptibility patterns of aerobic and facultative gram-negative bacilli causing intraabdominal infections: Results from the SMART studies 2003-2007. Rev. Esp. Quimioter, 21: 166-173. http://seq.es/seq/0214-3429/21/3/guembe.pdf

Hasper, D., J.C. Schefold and D.C. Baumgart, 2009. Management of severe abdominal infections. Recent Pat. Antiinfect Drug. Discov., 4: 57-65. PMID: 19149697

Jouini, A., K.B. Slama, Y. Saenz, N. Klibi and D. Costa et al., 2009. Detection of multipleantimicrobial resistance and characterization of the implicated genes in Escherichia coli isolates from foods of animal origin in Tunis. J. Food. Prot., 72: 1082-1088. PMID: 19517738

Koksal, F., K. Ak, O. Kucukbasmaci and M. Samasti, 2009. Prevalence and antimicrobial resistance patterns of extended-spectrum $\beta$-lactamaseproducing escherichia coli and Klebsiella pneumoniae isolated from blood cultures in an istanbul university hospital. Chemotherapy, 55: 293-297. DOI: $10.1159 / 000224657$

Kroenke, K., 1985. Polypharmacy: Causes, consequences and cure. Am. J. Med., 79: 149-152. PMID: 4025372
Laterre, P.F., F. Colardyn, M. Delmee, J. De Waele and J.C. Legrand et al., 2006. Antimicrobial therapy for intra-abdominal infections: Guidelines from the Infectious Disease Advisory Board (IDAB). Acta Chir. Belg., 106: 2-21. PMID: 16612906

Marshall, J.C., 2004. Intra-abdominal infections. Microbes Infect., 6: 1015-1025. DOI: 10.1016/J.MICINF.2004.05.017

Mazuski, J.E., 2007. Antimicrobial treatment for intraabdominal infections. Exp. Opin. Pharmacother., 8: 2933-2945. DOI; 10.1517/14656566.8.17.2933

Mazuski, J.E., R.G. Sawyer, A.B. Nathens, J.T. DiPiro and M. Schein et al., 2002a. The Surgical infection society guidelines on antimicrobial therapy for intra-abdominal infections: Evidence for the recommendations. Surg. Infect., 3: 175-233. DOI: $10.1089 / 109629602761624180$

Mazuski, J.E., R.G. Sawyer, A.B. Nathens, J.T. DiPiro and M. Schein et al., 2002b. The surgical infection society guidelines on antimicrobial therapy for intra-abdominal infections: An executive summary. Surg. Infect., 3: 161-173. DOI: 10.1089/109629602761624171

Mesaros, N., P. Nordmann, P. Plesiat, M. RousselDelvallez and J. Van Eldere et al., 2007. Pseudomonas aeruginosa: Resistance and therapeutic options at the turn of the new millennium. Clin. Microbiol. Infect., 13: 560-578. DOI: $10.1111 / \mathrm{j} .1469-0691.2007 .01681 . \mathrm{x}$

Montravers, P., A. Lepape, L. Dubreuil, R. Gauzit and Y. Pean et al., 2009. Clinical and microbiological profiles of community-acquired and nosocomial intra-abdominal infections: Results of the French prospective, observational EBIIA study. J. Antimicrob. Chemother., 63: 785-794.DOI: 10.1093/jac/dkp005

Obritsch, M.D., D.N. Fish, R. M. Laren and R. Jung, 2005. Nosocomial infections due to multidrug-resistant Pseudomonas aeruginosa: Epidemiology and treatment options. Pharmacotherapy, 25: 13531364. DOI: 10.1592/phco.2005.25.10.1353

Onderdonk, A.B., R.L. Cisneros, R. Finberg, J.H. Crabb and D.L. Kasper, 1990. Animal model system for studying virulence of and host response to bacteroides fragilis. Rev. Infect. Dis., 12: S169-S177. PMID: 2406869

Paterson, D.L., F. Rossi, F. Baquero, P.R. Hsuehand G.L. Woods et al., 2005. In vitro susceptibilities of aerobic and facultative Gram-negative bacilli isolated from patients with intra-abdominal infections worldwide: The 2003 Study for Monitoring Antimicrobial Resistance Trends (SMART). J. Antimicrob. Chemother., 55: 965973. DOI: $10.1093 /$ jac/dki117 
Pieracci, F.M. and P.S. Barie, 2007a. Intra-abdominal infections. Curr. Opin. Crit. Care, 13: 440-449. DOI: 10.1097/MCC.0b013e32825a6720

Pieracci, F.M. and P.S. Barie, 2007b. Management of severe sepsis of abdominal origin. Scand. J. Surg., 96: 184-196. http://www.fimnet.fi/sjs/articles/SJS32007-184.pdf

Schito, G.C., K.G. Naber, H. Botto, J. Palou and T. Mazzei et al., 2009. The ARESC study: An international survey on the antimicrobial resistance of pathogens involved in uncomplicated urinary tract infections. Int. J. Antimicrob. Agents, 34: 407-413. DOI: 10.1016/J.IJANTIMICAG.2009.04.012

Gholamreza-Sepehri, G. and M.S. Meimandi, 2005. The quality of prescribing in general practice in Kerman, Iran. Int. J. Health Care Qual. Assur. Inc. Leadersh. Health Serv., 18: 353-360. PMID: 16167650

Smith, T.L., M.L. Pearson, K.R. Wilcox, C. Cruz and M.V. Lancaster et al., 1999. Emergence of vancomycin resistance in staphylococcus aureus. N. Engl. J. Med., 340: 493-501. http://content.nejm.org/cgi/reprint/340/7/493.pdf
Solomkin, J.S., J.E. Mazuski, E.J. Baron, R.G. Sawyer and A.B. Nathens et al., 2003. Guidelines for the selection of anti-infective agents for complicated intra-abdominal infections. Clin. Infect. Dis., 37: 997-1005. DOI: 10.1086/378702

Weigelt, J.A., 2007. Empiric treatment options in the management of complicated intra-abdominal infections. Cleve. Clin. J. Med., 74: 29-37. http://www.ccjm.org/content/74/Suppl_4/S29.long

Zapantis, A., M.K. Lacy, R.T. Horvat, D. Grauer and B.J. Barnes et al., 2005. Nationwide antibiogram analysis using NCCLS M39-a guidelines. J. Clin. Microbiol., 43: 2629-2634. DOI: 10.1128/JCM.43.6.2629-2634.2005 